\title{
Improving credit scoring by differentiating defaulter behaviour
}

\author{
Cristián Bravo ${ }^{1,3 *}$, Lyn C Thomas ${ }^{2}$ and Richard Weber ${ }^{3}$ \\ ${ }^{I}$ Departamento de Modelamiento y Gestión Industrial, Universidad de Talca, Curicó, Chile; ${ }^{2}$ Southampton \\ School of Management, University of Southampton. Highfield, Southampton, UK; and ${ }^{3}$ Department of Industrial \\ Engineering, Universidad de Chile, Santiago, Chile
}

\begin{abstract}
We present a methodology for improving credit scoring models by distinguishing two forms of rational behaviour of loan defaulters. It is common knowledge among practitioners that there are two types of defaulters, those who do not pay because of cash flow problems ('Can't Pay'), and those that do not pay because of lack of willingness to pay ('Won't Pay'). This work proposes to differentiate them using a game theory model that describes their behaviour. This separation of behaviours is represented by a set of constraints that form part of a semi-supervised constrained clustering algorithm, constructing a new target variable summarizing relevant future information. Within this approach the results of several supervised models are benchmarked, in which the models deliver the probability of belonging to one of these three new classes (good payers, 'Can't Pays', and 'Won't Pays'). The process improves classification accuracy significantly, and delivers strong insights regarding the behaviour of defaulters.
\end{abstract}

Journal of the Operational Research Society advance online publication, 7 May 2014; doi:10.1057/jors.2014.50

Keywords: credit scoring; statistics; domain-knowledge; constrained clustering; banking; game theory

\section{Introduction}

Credit scoring (Thomas et al, 2002) is one of the most widely known applications of statistical models and data mining, whose goal is to differentiate between customers who will pay back a given loan, and those who will not. Classifying borrowers into these two groups (defaulters and non-defaulters) has been the standard approach of credit scoring from its inception. Lately, there has been a rise in the number of statistical models that use economic analysis and game theory to better understand the behaviour of the relevant players in many applications. It has been used to improve manufacturing strategies (Wang, 2007), in credit card fraud detection (Vatsa et al, 2005), and in phishing detection for spam filtering (L'Huillier et al, 2009), to name a few. In this paper, we present a procedure that extends application credit scorecards by differentiating defaulters into two groups, those who default due to the lack of willingness to repay, and those who fail to pay because they do not have the capacity to do so.

The relationship between lenders and their borrowers has been studied by a number of authors modelling who are defaulters and what are the probabilities of default. For example in the wellknown work of Stiglitz and Weiss (1981), the authors imposed conditions on the rationality of the players, which produce an adverse selection game in the credit granting process. The

\footnotetext{
*Correspondence: Cristián Bravo, Department of Modelling and Industrial Management, Universidad de Talca. Camino a Los Niches Km. 1, Curicó, 3344158, Chile.
}

importance of collateral as a way of selecting customers was also studied by Wette (1983). Both papers point out that the drivers for the decisions of whether to request a loan and whether to grant the request include the amount of the loan and the collateral offered.

More recent works have focused more on reasons and characteristics of default. Alary and Gollier (2004), using collateral and amount lent as their main decision variables, emphasized the moral hazard problem that lenders faced, and showed that under certain conditions customers will default strategically. Moffat (2005) used hurdle models to model default, and the extent of it, also finding different levels or intensities of default. Block-Lieb and Janger (2006) suggested that the expectation that borrowers will be fully rational is not true in some cases, and that it is not reasonable to assume that all customers behave strategically. The work of Guiso et al (2010) also focused on the reasons that drive default, and found that there are segments of the population that default strategically, and that such behaviour is driven by economic and moral variables. Finally, differences in default and the characteristics of defaulters have also been studied in small business lending, with Lin et al (2011) defining four different types of default depending on the financial conditions of borrowers.

The literature agrees that there are different reasons for defaulting, even though credit scoring aggregates these different reasons into just one default class, which is normally defined as being 90 days in arrears (Thomas, 2000). Then one usually estimates the risk of this happening in the next year. Our 
proposal is to differentiate between two types of default, for both willingness and capacity to repay, by using first economic modelling, and then semi-supervised clustering. Then it is possible to build a scoring system using supervised techniques such as logistic regression or neural networks to predict whether a borrower will default for each specific reason.

This paper is divided as follows: The next section presents a game theory model that determines which combination of collateral, loan rate charged, and borrower/lender characteristics lead to loans being given, but then defaulted upon. The borrowers are assumed to belong to one of two groups; those who are unwilling to repay the loan (colloquially referred to as 'Won't Pays') and those who want to repay but may default because they do not have the capacity to repay (colloquially called 'Can't Pays').

The subsequent part of the paper shows how the behaviours described by the economic model can be used as input for a semi-supervised clustering procedure, which separates borrowers into one of the two classes. In order to demonstrate the usefulness of the proposed model, the resulting procedure is applied to a dataset of actual loans in the 'Validation and experimental results' section. In it, the clustering procedure is conducted to find which are the 'Won't Pay' and which the 'Can't Pay' clusters. Then, two supervised learning models (neural networks and multinomial logistic regression) are used on the data using the cluster labels as targets. The results of the supervised models are benchmarked against the classical logistic regression model based on the whole, un-clustered, population to obtain an overall estimation of the borrowers' credit risk, focusing on both the gains in accuracy and the knowledge obtained. Finally, the conclusions extracted from building such a model are outlined.

\section{Game theory model of the loan granting process}

Throughout this paper we will assume there is a set of $N$ borrowers that were granted a loan. Each loan is described by a set $V$ of different variables, stored in database $X \subset \mathbb{R}^{|V|}$, representing the different characteristics of the loan, the borrower, and the evolution of the loan. There is also an outcome variable, given by $d_{i} \in\{0,1\}$, associated with each $x_{i} \in X$ $(i \in\{1, \ldots, N\})$, indicating whether borrower $i$ defaulted on that loan or not. Only the characteristics in $V$, which describe data known before the loan was granted, can be used for classification, which we denote as $V_{\text {past }} \subset V$, forming a dataset $X_{\text {past. }}$. The remaining characteristics, $V_{\text {future }}=V \backslash V_{\text {past }}$ and the corresponding dataset $X_{\text {future }}$, consist of information that can only be used for determining whether and for what reasons the borrower has defaulted. The database will consist of different applicants, with one loan per applicant, as is common in application scoring.

In general, a game is defined by its players, their strategies $s_{i}$, and the payoffs $u_{i}\left(s_{i}, s_{-i}\right)$ of playing the various strategies in the competitive or adversarial setting (Fudenberg and Tirole, 1991), where $s_{-i}$ represents the strategies played by all other players except $i$. In the model of the loan granting process presented in this work, the first player, $L$, is the lender who wants to maximize the profit of lending to $N$ potential borrowers over two time periods.

The borrowers can be of two types, $C$ or $W$, where $C$ s are the 'Can't Pays' who are willing to pay back the loan they received provided they are able, and so will only default because of the occurrence of an external shock that can occur with probability $q$, that can be, for example, job loss of the borrower. Considering that we will perform classification further on, we will refer to the types of borrowers as either types or classes. Class $W$ corresponds to the 'Won't Pays' who are unwilling to pay back the loan even if they can afford to do so, and so presumably took the loan with no intention of paying it back. We can also identify the good payers (class $P$ ) as members of class $C$ that are not subject to an external shock, and thus were able to repay the loan.

The mechanism for granting loans will be the following: in the first period borrower $i \in\{1, \ldots, N\}$ can ask for a loan $a_{i 1}$, and the lender can agree to or refuse this request, where $y_{i 1}=1$ is accepting the loan request and $y_{i 1}=0$ is refusing it. The loan is paid at the next period together with the interest, at an interest rate of $r$, charged on the loan. The lender could also ask for collateral $C_{i 1}$ from the borrower $i$ to secure the loan, where $C_{i 1}$ could be, for example, the deed to the house on which a mortgage is taken out; or the lender could grant the loan without collateral (an unsecured loan, ie $C_{i 1}=0$ ). If the loan is not repaid, the lender will sell the collateral but will only get an amount $\alpha C_{i 1}$ back, with $\alpha \in[0,1]$, so $(1-\alpha)$ is the so-called 'haircut' on the collateral. Borrowers of type $C$ will not be able to repay if they have had a shock to their cash flow during the period, while those of type $W$ will not pay no matter what their cash flow is.

We assume that a shock leads to default, and if no shock occurs then borrowers from class $C$ will always repay. On the basis of this assumption we can focus our analysis of the decision process just on the marginal income that the loan brings to the borrowers. Our assumption occurs in segments in which income is concentrated in a narrow range, a common occurrence for micro-entrepreneurs and low-income consumers, as we will show in the experimental section below.

If the loan is repaid, the borrower can repeat the process in the second period asking for a loan of value $a_{i 2}$ which, if it is given, will correspond to decision $y_{i 2}=1$ by the lender, as opposed to $y_{i 2}=0$ if refused. The interest rate remains $r$, but the collateral this time would be $C_{i 2}$. If the loan is not repaid in the first period, the borrower will not be granted a new loan in the second period. If the first loan was not granted, it is not reasonable that this would be different in the second period, so it will not be granted as well. The time dependence of the players' utility is given by discount rates defined to be $\delta_{L}, \delta_{C}$, and $\delta_{W}$ for the lender and the two types of borrowers, respectively.

If we do not restrict the total amount of money that the lender has available, then this procedure can be considered as a series of two-player games between the lender and each of the individual borrowers. In this case, without loss of notation, we 
will ignore the index $i$ of each borrower. The payoff of the two types of borrowers and the lender, $u_{C}, u_{W}$, and $u_{L}$, will depend on the strategies $s=\left(y_{1}, y_{2}, a_{C 1}, a_{C 2}, a_{W 1}\right)$ chosen, together with the a priori probability that the borrower is in class $C$, represented by $\theta \in(0,1)$. The lender does not know which type the current borrower is. This is private information that the borrower has, so this is a game with incomplete information.

The expected utility of the borrowers is the net present value of the loans they receive, according to their type:

$$
\begin{aligned}
u_{C}(s)= & y_{1}\left(a_{C 1}-q \delta_{C} C_{1}-(1-q) \delta_{C}(1+r) a_{C 1}\right) \\
+ & (1-q) \delta_{C} y_{1} y_{2}\left(a_{C 2}-q \delta_{C} C_{2}\right. \\
- & \left.(1-q) \delta_{C}(1+r) a_{C 2}\right) \\
& u_{W}(s)=y_{1} a_{W 1}-y_{1} \delta_{W} C_{1}
\end{aligned}
$$

The utility of the lender is the expected value of the returns from the loans:

$$
\begin{aligned}
u_{L}(s)= & y_{1}(1-\theta)\left(-a_{W 1}+\delta_{L} \alpha C_{1}\right) \\
& +y_{1} \theta\left(-a_{C 1}+q \delta_{L} \alpha C_{1}+(1-q) \delta_{L}(1+r) a_{C 1}\right) \\
& +\theta(1-q) \delta_{L} y_{1} y_{2}\left(-a_{C 2}+q \delta_{L} \alpha C_{2}\right. \\
& \left.+(1-q) \delta_{L}(1+r) a_{C 2}\right)
\end{aligned}
$$

To derive the set of conditions that the player must satisfy, we will create a set of constraints that account for the individual rationality of the players given what was observed to occur, that is, we will demand that the best possible choice is to request and grant the requested loans.

We will assume one condition for this setting: that the strategy of granting or requesting two loans (or one in case of borrowers in class $W$ ) is individually rational. Thus, requesting and granting two loans has a higher utility compared with not participating or to just requesting a loan in one period for class $C$, which would bring a marginal utility equal to zero.

For the lender, the conditions are:

$$
\begin{array}{cc}
u_{L}\left(1,1, C_{1}, C_{2}, s_{-i}^{\prime}\right) \geqslant u_{L}\left(1,0, C_{1}, 0, s_{-i}^{\prime}\right) & \forall C_{1}, C_{2} \\
u_{L}\left(1,1, C_{1}, C_{2}, s_{-i}^{\prime}\right) \geqslant u_{L}\left(0,0,0,0, s_{-i}^{\prime}\right) & \forall C_{1}, C_{2}
\end{array}
$$

The right-hand side (RHS) of (4) is equal to:

$$
\begin{aligned}
u_{L}\left(1,0, C_{1}, C_{2}, s_{-i}^{\prime}\right)= & \theta a_{C 1}\left(-1+\delta_{L}(1-q)(1+r)\right) \\
& -(1-\theta) a_{W 1}+\delta_{L} \alpha C_{1}(1-\theta+\theta q)
\end{aligned}
$$

And so (4) is equivalent to:

$$
a_{C 2}\left(-1+\delta_{L}(1-q)(1+r)\right)+(1-q) q \delta_{L} \alpha C_{2} \geqslant 0
$$

(5) is equivalent to:

$$
\begin{aligned}
\theta & \left(a_{C 1}+a_{C 2} \delta_{L}(1-q)\right)\left(-1+\delta_{L}(1-q)(1+r)\right) \\
& -(1-\theta) a_{W 1}+C_{1} \alpha \delta_{L}(1-\theta(1-q)) \\
& +\theta(1-q) q \delta_{L}^{2} \alpha C_{2} \geqslant 0
\end{aligned}
$$

To obtain the solution space, the same analysis has to be carried out for each borrower. For borrowers of class $C$, the inequalities that must be satisfied are:

$$
\begin{gathered}
u_{C}\left(a_{C 1}, a_{C 2}, s_{-i}^{\prime}\right) \geqslant u_{C}\left(a_{C 1}, 0, s_{-i}^{\prime}\right) \\
u_{C}\left(a_{C 1}, a_{C 2}, s_{-i}^{\prime}\right) \geqslant u_{C}\left(0,0, s_{-i}^{\prime}\right)
\end{gathered}
$$

Note that this assumes that $y_{1}=1$ and $y_{2}=1$, that is inequalities (4) and (5) are satisfied. This is reasonable because every other decision implies utilities of $u_{C}\left(0,0, s^{\prime}{ }_{-i}\right)$, that is, no loans are granted.

The RHS value from (10) equals zero, so the borrower only asks for a loan if she or he receives positive utility. Incorporating that into (10) implies:

$$
\begin{aligned}
& a_{C 1}\left(1-\delta_{C}(1-q)(1+r)\right) \\
& \quad+\delta_{C} a_{C 2}(1-q)\left(1-\delta_{C}(1-q)(1+r)\right) \\
& \quad-\delta_{C} q C_{1}-\delta_{C}^{2}(1-q) q C_{2} \geqslant 0
\end{aligned}
$$

Condition (9) assumes that the expected utility that arises from applying for a second loan must be positive, so that:

$$
a_{C 2} \geqslant \frac{\delta_{C} q}{1-\delta_{C}(1-q)(1+r)} C_{2}
$$

Finally, borrowers of class $W$ will apply for a loan if their respective utility is greater than the discounted collateral they would lose if they received the loan:

$$
a_{W 1} \geqslant \delta_{W} C_{1}
$$

The set of inequalities (7), (8), (11), (12), and (13) creates a behaviour space. This set of inequalities can be interpreted as a set of constraints that will be used to separate the behaviour of the defaulters. A defaulter of class $C$ desires a second loan even though this will be refused since he or she will have defaulted, while a defaulter of class $W$ does not expect a second loan. Given this set of constraints, it is now possible to actually differentiate the defaulters: we can obtain two groups, each one with cases that are similar (in variance) and that satisfy the proposed constraints.

Some other interesting results that arise from the proposed model are as follows:

- Since the left-hand side (LHS) of (5) is increasing in $C_{1}, C_{2}$, and $\delta_{L}$ the collaterals are an incentive for the lender to grant loans. Similarly, a lower expected return on capital, and so a higher discount factor $\delta_{L}$, increases the chance the loan will be given.

- (12) and (13) show that borrowers might accept a loan even if the collateral demanded is of greater value than the loan itself. Such event is observed in real life, for example, when a loan to finance a percentage of a car is secured by the value of the car in full.

- A necessary condition for the set defined by the constraints to be non-empty is that $\delta_{W}<\delta_{C}<\delta_{L}$. This is reasonable since people who need to borrow have lower discount factors than the lending organizations, and borrowers in class $W$ are 
assumed to be more short-sighted than the other types of borrowers. A high value of $\delta_{W}$ would also result in that group being limited to very high loan amounts (relative to the collateral), or to borrowers with no collateral.

We propose a learning model that incorporates such restrictions in the next section.

\section{Constrained clustering and semi-supervised methods}

Constrained clustering (Basu et al, 2008) is a semi-supervised approach to obtaining segments of a dataset incorporating certain restrictions that must be fulfilled by the members of the cluster, the members of different clusters, or the general structure of the clusters. The term 'semi-supervised' refers to the incorporation of knowledge that is not directly present in the data - or that is known only for a limited number of cases-in order to improve the results on the whole domain. In this particular case, restrictions are added to a clustering procedure so that the objective is not only to minimize intra-cluster variance, but also to satisfy a set of conditions for each member, or each cluster. The methodology has been applied successfully in several fields, ranging from signal processing (Levy and Sandler, 2008), epidemiology (Patil et al, 2006), to OR applications (Bard and Jarrah, 2009).

There are two different approaches for constrained clustering, as noted by Davidson and Ravi (2005), and both are based on the concept of 'Must-Link' and 'Cannot-Link' constraints. The first set of restrictions indicate that two elements must be in the same cluster, whereas the second set prohibits the presence of two elements in the same cluster. The methods differ in the role of restrictions: in the first case the algorithm satisfies an objective or distance function using the information from the constraints. The best-known application of this work is by Basu et al (2004). In the second case the constraints simply limit the presence of elements in the same cluster. Our work uses the second approach, with an adjustment that adds some complexity: the elements in one cluster must satisfy the restrictions against most of the elements of the other cluster. There are algorithms that solve this particular problem, such as the Constrained Clustering with Filtering (CCF) algorithm introduced by Bravo and Weber (2011), which will be used in this paper.

\section{A constrained clustering model to differentiate defaulters}

The constraints obtained from the economic assumptions from previous sections can now be used to design a new objective variable using semi-supervised methods. The overview of the process is as follows:

1. Select defaulters from the database $X$, and describe them using only the information in $X_{\text {future }}$, composed of variables in $V_{\text {future }}$, that is, variables collected after the loan has been granted.
2. Cluster elements into two groups, one for each type of defaulter $(C$ and $W$ ) using the CCF algorithm with dataset $X_{\text {future }}$ as input. The constraints defined in the previous section are extended now to the whole cluster, requiring that most cases in the cluster assigned to class $W$ have to satisfy their own condition, Equation (13), and that elements in the cluster assigned to class $C$ must satisfy constraints (7) and (10). Finally there is a cross-cluster constraint, given by the lender condition (8), which must be satisfied by 'most' pairs of elements in different clusters.

3. With the elements clustered, the new objective variable extends the default variable to the new case when there are three different cases: good payers, defaulters in class $C$, and defaulters in class $W$.

The exact constrained clustering problem to be solved considers two groups with centroids given by $c_{k}, k=\{1,2\}$ and a binary variable $m_{i}$ for each $i \in\{1, \ldots, N\}$ that represents the class of the borrower ( 1 for class $C, 0$ for class $W$ ). Since the value of the second loan and the second collateral are not known, we will assume they are a fraction of the original value requested $\left(f_{C r}\right.$ and $f_{C o l}$, as explained below. Since now each borrower has his or her own variables, we will refer to the amount borrowed as $a_{i} \in x_{i}$, to the collateral given for the first loan as $C_{i} \in x_{i}$ and finally to the interest rate paid as $r_{i} \in x_{i}$, with $x_{i}$ the vector of variables for each borrower/loan

The problem is then to solve the following optimization problem, adapted from the formulation presented by Dogan and Guzelis (2006):

$\min _{m_{1}, m_{2}, c_{1}, c_{2}, \max _{W}, \min _{C}} \sum_{i=1}^{N} m_{i}\left\|x_{i}-c_{1}\right\|^{2}+\left(1-m_{i}\right)\left\|x_{i}-c_{2}\right\|^{2}$

s.t. $\quad \min _{C} \geqslant(1-\theta) a_{i} \quad \forall i \mid m_{i}=0$

$$
x_{i} \geqslant \delta_{W} C_{i} \quad \forall i \mid m_{i}=0
$$$$
0 \leqslant a_{i}\left(1-\delta_{C}(1-q)\left(1+r_{i}\right)\right)
$$$$
\times\left(1+\delta_{C} f_{C r}(1-q)\right)
$$$$
-C_{i} \delta_{C} q\left(1+\delta_{C} f_{C o}\right) \quad \forall i \mid m_{i}=1
$$$$
0 \geqslant \theta a_{i}\left(-1+\delta_{L}(1-q)\left(1+r_{i}\right)\right)
$$$$
\times\left(1+f_{C r} \delta_{L}(1-q)\right)
$$$$
+C_{i} \alpha \delta_{L}\left(1-\theta(1-q) q \delta_{L} f_{C o}\right)
$$$$
-(1-\theta) \max _{W} \quad \forall i \mid m_{i}=1
$$

$\min _{C} \leqslant \theta a_{i}\left(-1+\delta_{L}(1-q)(1+r)\right)$

$\times\left(1+\delta_{L} f_{C r}(1-q)\right)$

$+C_{i} \alpha \delta_{L}(1-\theta(1-q)$

$$
\left.+\theta f_{\text {Col }}(1-q) q \delta_{L}\right) \quad \forall i \mid m_{i}=1
$$




$$
\begin{aligned}
\max _{W} & \geqslant a_{i} \quad \forall i \mid m_{i}=0 \\
c_{1} & =\frac{\sum_{i=1}^{N} m_{i} x_{i}}{\sum_{i=1}^{N} m_{i}} \\
c_{2} & =\frac{\sum_{i=1}^{N}\left(1-m_{i}\right) x_{i}}{N-\sum_{i=1}^{N} m_{i}} \\
m_{i} & \in\{0,1\} \quad \forall i \in\{1, \ldots, N\}
\end{aligned}
$$

Expression (14) represents a quadratic objective function with integer variables. The constraints (15) depend directly on the cluster in which the elements are present, so an extensive expression (one that does not include the $m_{i}$ parameters in the description) would have to include $N^{2}$ constraints, one for each pair of elements, turning the problem intractable for large databases.

The CCF algorithm follows a similar methodology as the K-Means algorithm, extending the procedure for constrained problems. In each iteration the set of constraints for each element is checked, that is, if the element is in class $C$, then Equations (11) and (12) are evaluated for the element itself, and Equation (8) is evaluated against the extreme values arising from the elements currently in the cluster assigned to class $W$. In case the conditions are not fulfilled, the element is moved from its original cluster, and if that does not solve the issue, the elements with extreme values in both clusters are removed from the analysis and the process is repeated. The algorithm continues until the violations are below a threshold and the centroid values do not move more than a given tolerance.

The elimination of cases with extreme values in each iteration relaxes the problem. The elements in each cluster have to satisfy the constraints against most of the elements in the other cluster, and this is accomplished by eliminating a small number of extreme cases in each iteration and ensuring that all remaining cases satisfy the constraints. In the end, since the final cluster for each element is determined only by proximity to centroids $c_{1}$ and $c_{2}$, it is possible to re-assign even the cases that were eliminated from the clustering procedure.

One of the open questions that remain is what are the possible values of the parameters required to estimate the model. We propose the following values, which correspond to realistic measures in credit risk:

- $\theta$ : This parameter represents the a priori probability of a customer being in the class 'Can't Pay'. The lender can estimate this value from historical data, using two different approaches: In the experimental part of this paper the proportion of defaulters among all defaulters that made a payment until up to 2 years after the default occurred was used as an approximation of $\theta$. This is based on the assumption that defaulters of type $W$ pay back very little of the loan or nothing at all. The second method is using the number of instalments paid, since defaulters in class $W$ are expected to repay very little, if nothing at all, of the loan. In this case the proportion of borrowers who default in the first instalments of the loan are an approximation of the value $1-\theta$. Note that this value would have to be adjusted to account for borrowers in class $C$ that suffer a liquidity shock in the first few months.

- $q$ : This parameter represents the chance that a borrower receives a shock to his or her income, and so is forced to default. This value could correspond to the long-term default rate for loans, because it is expected that in the long term, most of the 'Won't Pay' borrowers are filtered from the database. Another option (and the one used in the experimental results section) is to discount the observed default rate $(D R)$ by a small amount, in order to include the unobserved segment. A possible value would be $q=(D R \cdot(1-\theta) / 2)$.

- $\alpha$ : The expected recovery to be extracted from the collaterals is a known value to credit granters, with values commonly between $40 \%$ and $60 \%$ of the collateral value (see for example Yamashita and Yoshiba, 2010 or Jokivuolle and Peura, 2000).

- $\delta_{L}$ : The value of the discount factor for a company should be a known value, for example if the expected internal return rate for the fiscal year is $\tau$, then the company's discount factor would be $1 /(1+\tau)$.

- $\delta_{C}$ and $\delta_{W}$ : The value of the discount factor for the customers is more difficult to determine. Individual discount factors have been studied on several occasions with widely different results. For example Burks et al (2008), Chabris et al (2008), and Green et al (1994) report discount rates ranging from 0.1 to 0.9 depending on several factors, although these studies do not focus on financial decisions. The well-known paper of Benzion et al (1989) gives discount factors depending on the financial amount at risk that ranges from 0.2 to 0.75 , with a strong dependence on both the amount at risk and the duration of the loan. We can imply from such studies that there is a time-value of money, which allows lending money to occur, but that the exact value of such discount factor is hard to measure. We propose an exogenous measure, which limits the range of choices that a borrower has for such rate. In many countries there is a legal limit for the annual rate that can be charged by lenders, and this limit can be justified as a constraint on an irrational behaviour from the borrowers facing an extreme situation. Low tomiddle-income borrowers intuitively understand these limits and make rational decisions without them (Littwin, 2007). The maximum interest rate allowed for loans, which represents the maximum discount rate that any customer is legally bound to pay, can then be a good proxy of the maximum rate a rational borrower would accept. We proceed with the modelling process fixing that value for $\delta_{C}$. As for $\delta_{W}$, the value has to be lower than that associated with customers of class $C$, because their demand for the loan is more immediate. We study a range of values in $\left[0, \delta_{C}\right]$ in the 'Validation and experimental results' section. 
- Values for the second loan: Two of the parameters that must be decided are the values for the second loan and the second collateral. Since defaulters were not allowed to take a new loan, these values are unknown, but they are known for the customers who successfully paid back their loans. Good proxies for these values are the proportions between the amounts that were requested/granted by returning borrowers, that is, the average value of $f_{C r}=$ (Second_Amount/First_Amount $)$ and $f_{C o l}=($ Second_Collateral/First_Collateral $)$ calculated for borrowers who successfully returned granted loans and received new ones.

\section{Validation and experimental results}

In this section we present the results of applying the proposed approach to a dataset of loans granted at a financial institution. First the dataset is introduced, then we present the procedure of constrained clustering on the defaulters from this dataset. In the section 'Sensitivity analysis and parameter selection' we perform those analyses. Finally, we discuss in detail the obtained results.

\section{Available dataset}

A dataset consisting of 97254 loans granted to mass-market, low to middle-income independent borrowers (with a monthly income ranging from USD 300 to USD 2000) was used. The database originates from a Chilean public organization, and comprises an 11-year period, from 1997 to 2007. The dataset has a default rate of $25.2 \%$, with each loan described by 25 different variables. One of the main characteristics of the dataset is that the organization almost never rejected a loan: its reject rate was just $2 \%$, so there is little need to use reject inference. This extremely low rejection rate also explains the very high default rate, as will be reflected in the composition of the cases in classes $C$ and $W$.

The variables available for classification, that is the ones obtained before granting loans are as follows:

- Economic activity: The sector of the economy in which the customer is involved (through his/her job or company). The large number of sectors was clustered to improve interpretation of the variable, mapping the 47 different sectors to three larger groups (Activity_A, Activity_B, and Activity_C), and the last one was selected as a reference category.

- Ownership of housing: This shows if the customers own, rent, or hold other types of agreements on their current home. Four classes are recognized: Owner, Tenant, Share-Tenant (Share), or other types. The class 'Others' is used as a reference.

- Number of properties: The number of properties the borrower possesses. The variable was divided into three categories: No properties, one property, or two or more properties, which was used as a reference category.

- Region of country: Division of the country into three regions, one of them (arbitrary) used as a reference.
- With guarantor: Whether the customer has a guarantor for the loan or not.

- Length of loan: The length of the loan requested by the borrower. This number is determined by the customer, with the company simply granting the loan or refusing it, so it is not susceptible to manipulation. The durations of the loans are between 1 and 12 months.

- Age: The age of the customer in years.

The previously presented variables describe the customer at the moment of requesting the loan. On the other side, the variables that describe the actual evolution of the loan are as follows:

- Collaterals: The collaterals are described by two variables. The first is a dummy variable that describes whether the customer had to give collateral on the loan (With_Collaterals), and the value of the collateral (Value_Collateral_UF). The latter is given in 'development units' (Unidades de Fomento, referred to as UF for their acronym in Spanish), the Chilean inflation-indexed unit, that is equivalent to roughly 46 USD.

- Amount and rate: The amount of the loan, in UF, and the total annual interest rate charged for the loan.

- Days in arrears before defaulting (Days_Arrear): The total number of days the instalments of the loan were in arrears before defaulting. For example, if a loan defaulted in the fourth instalment, and the first was paid 10 days late, the second on time, and the third 45 days late, the variable has a value of 55 .

- Cancellations: Sometimes the institution will cancel the payment of penalties and excess interest that arises from arrears, upon agreement that the next instalment is paid on time, or that a renegotiation is performed. This event is summarized into two different variables, considering the number of times this happened in the lifetime of the loan (Num_Cond), and the amount that was reduced (Amount_Cond). In addition, if some of the interest due to be paid is also discounted from the instalments, this value is reported in the variable Interest_Low.

- Extensions: Sometimes the company will extend the period of an instalment for 30 days or a similar span of time, subject to adjustment in the amount owed. The number of times a customer applies for this appears in variable Num_Post, and the amount adjusted appears in Amount_Adjust, and, since the adjustment can be positive or negative, the total amount of negative adjustments is incorporated into Negative_Adj.

\section{Clustering procedure}

For the clustering procedure, the parameters used, their values, and their origin are shown in Table 1.

The process is run using the 24576 customers flagged as defaulters, normalizing the dataset with all the future variables. The results consist of two clusters, each one 
Table 1 Parameters selected for clustering experiment

\begin{tabular}{lll}
\hline Parameters & Value & Origin \\
\hline$q$ & 0.130 & Adjusted default rate \\
$\theta$ & 0.550 & Estimated from historical return data \\
$\alpha$ & 0.600 & Range [0.4, 0.6] \\
$\delta_{L}$ & 0.935 & Company factor-10\% inflation \\
$\delta_{C}$ & 0.625 & $55 \%$ maximum annual rate (Central Bank) \\
$\delta_{W}$ & 0.500 & From sensitivity analysis \\
$f_{C r}$ & 1.320 & Examples from good payers \\
$f_{\text {Col }}$ & 2.470 & Examples from good payers \\
\hline
\end{tabular}

Table 2 De-normalized results of semi-supervised clustering

\begin{tabular}{lrr}
\hline Variable & Class C & Class W \\
\hline With_Collaterals & 0.40 & 0.33 \\
Amount & 48.67 & 12.89 \\
Days_Arrear & 882.53 & 708.61 \\
Num_Cond & 0.27 & 0.37 \\
Num_Post & 0.84 & 0.43 \\
Num_Reneg & 0.71 & 0.43 \\
Amount_Adjunt & -0.26 & -0.07 \\
Amount_Cond & 4.02 & 2.35 \\
Negative_Adj & 0.38 & 0.08 \\
Interest_Low & 1.18 & 0.27 \\
Payments & 2.86 & 2.37 \\
Value_Coll_UF & 58.93 & 6.59 \\
Rate & 1.10 & 1.10 \\
\hline
\end{tabular}

corresponding with a class, either $C$ or $W$. In the first cluster there are 4762 borrowers, which are assigned to Class $C$, while the second cluster has 19814 borrowers, which are assigned to class $W$. The centroids for each class are shown in Table 2. The variables associated with collaterals have a greater impact in differentiating the clusters, even though the difference in the percentage of customers with collaterals is not huge (40\% versus $33 \%$ ). The value of the collaterals for class $C$ is almost nine times greater than that in class $W$. Since the percentage of borrowers with collaterals are relatively balanced between the two clusters, this result shows that it is not whether a loan is secured or not what differentiate defaulters, but what the value of the collateral is, which is an interesting result.

Customers in class $C$ request a far larger amount for their loans, which would be consistent with a default based on the capacity to repay. Considering the total number of days in arrears, class $C$ accumulates almost 100 days more than class $W$ before defaulting, indicating that they make a greater effort to pay back the loans than customers in class $W$ do. Also, they apply for a larger number of renegotiations $(0.71$ per customer on average), get greater adjustments and debt relieves, and are more susceptible to receive a discount on their interests due (1.18 UF per customer on average, versus 0.27 ). The values of the variables suggest that there are, indeed, different behaviours detected.
Table 3 Percentage of cases in class $W$ depending on parameters $\alpha$ and $\delta_{W}$

\begin{tabular}{lrrrrr}
\hline$\delta_{W} / \alpha$ & 0.40 & 0.45 & 0.50 & 0.55 & 0.60 \\
\hline 0.0 & $100.00 \%$ & $100.00 \%$ & $100.00 \%$ & $100.00 \%$ & $100.00 \%$ \\
0.1 & $100.00 \%$ & $100.00 \%$ & $100.00 \%$ & $100.00 \%$ & $100.00 \%$ \\
0.2 & $100.00 \%$ & $100.00 \%$ & $100.00 \%$ & $100.00 \%$ & $100.00 \%$ \\
0.3 & $86.17 \%$ & $85.08 \%$ & $80.14 \%$ & $86.17 \%$ & $81.13 \%$ \\
0.4 & $74.52 \%$ & $74.09 \%$ & $74.32 \%$ & $75.09 \%$ & $75.06 \%$ \\
0.5 & $79.82 \%$ & $74.56 \%$ & $72.14 \%$ & $71.92 \%$ & $70.79 \%$ \\
0.6 & $77.99 \%$ & $78.78 \%$ & $78.14 \%$ & $69.51 \%$ & $69.58 \%$ \\
\hline
\end{tabular}

\section{Sensitivity analysis and parameter selection}

In order to analyse the sensitivity of the clustering parameters, the procedure was run using several values of the parameters $\left(\delta_{W}, \alpha\right)$. The value of $\delta_{W}$ was tested in the range $[0,0.6]$, following the restrictions presented in the previous section, and $\alpha$ was simultaneously tested in the range $[0.4,0.6]$. Parameter $\alpha$ was varied in steps of 0.05 , while parameter $\delta_{W}$ was varied in steps of 0.1 .

Table 3 presents the obtained results, and shows that when the discount factor for the 'Won't Pays' $\left(\delta_{W}\right)$ is low, then the model assigns a large number of cases to that class. This is reasonable as a low value in the discount factor implies a more relaxed constraint, so it is easier to satisfy the restrictions for that class. Of greater interest is that this dependency presents very little variation when varying parameter $\alpha$ for all values except $\delta_{W}=0.6$, which seems to imply that after a certain threshold, the restrictions tend to balance and more cases can be assigned to class $C$. Higher values of parameter $\alpha$ relax the constraints applied to class $C$, but this effect was not strong until the discount factor $\delta_{W}$ reached a value of 0.5 .

Considering the results obtained, the values $\delta_{W}=0.5$ and $\alpha=0.6$ are selected. This is done because the number of elements in the cluster assigned to class $W$ decreases for increasing values of $\delta_{W}$ within the interval $(0.5,0.6)$. Outside this interval the respective number does not vary notably. This indicates that the value of $\delta_{W}=0.5$ is a critical value for the discount factor. With $\delta_{W}$ fixed at 0.5 , a value of $\alpha=0.6$ presents approximately 6600 cases in class $C$, which is a sufficient number to ensure a valid statistical model when applying supervised models for classification.

Of note is the large percentage of cases assigned to class $W$ in comparison to class $C$. This suggests that willingness to repay, not capacity to repay, is the most important factor that determines default for the analysed data. It is to be believed that this imbalance might be caused by three different factors: first, since the rejection rate of the organization is extremely low, then it follows that the organization possessed poor evaluation standards previous to the implementation of a scoring system, as we know it is the case. Bravo et al (2013) evaluated the impact of implementing a scoring system for this organization. Second, the clustering methodology itself has an 
impact in the imbalance of the classes, since class $C$ has an additional constraint that might make it harder for a case to be assigned to it, but this can be easily measured by the sensibility analysis performed, as we have shown in this section. A final factor is that loans tend to default at a constant, higher rate, during the first months of repayment, as shown for example in Baesens et al (2005) and this rate flattens at the final stages of the loan. Such cases of early default will be assigned to class $W$ but are actually class $C$ cases who have defaulted early. Combined with the second factor this will increase the number of cases in class $W$.

\section{Classification results}

In order to show the potential of our proposed approach we apply two different procedures to the same dataset. First, we apply standard logistic regression without differentiating defaulters, and then we apply two methods for classification with three classes $(C, W$, and $P$ ), namely multinomial regression and a feed-forward neural network with a Multi-layer Perceptron architecture.

Multinomial logistic regression (Hosmer and Lemeshow, 2000 ) is the natural extension of the binomial logistic regression model, which in turn is the most widely used technique for building credit scorecards, as noted by Anderson (2007); Siddiqi (2006); Thomas et al (2002). The method uses only two logistic regressions. The first compares classes $C$ and $W$ and leads to coefficients $\beta_{C}$ and the second compares $P$ and $W$ and leads to coefficients $\beta_{P}$. The classification function for each class $k, k \in\{P, C\}$ is then:

$$
p_{k}(x)=\frac{\exp \left(\beta_{0}^{k}+\sum_{j=1}^{\left|V_{\text {past }}\right|} \beta_{j}^{k} x_{j}\right)}{1+\sum_{k^{\prime} \in\{P, C\}} \exp \left(\beta_{0}^{k^{\prime}}+\sum_{j=1}^{\left|V_{\text {past }}\right|} \beta_{j}^{k^{\prime}} x_{j}\right)}
$$

and for class $W$ the classification function is:

$$
p_{k}(x)=\frac{1}{1+\sum_{k^{\prime} \in\{P, C\}} \exp \left(\beta_{0}^{k^{\prime}}+\sum_{j=1}^{\left|V_{\text {past }}\right|} \beta_{j}^{k^{\prime}} x_{j}\right)}
$$

The second multinomial model used is feed-forward neural networks, judged to be the most accurate procedure for credit scoring according to Baesens et al (2003). Neural networks are known black-boxes, but several adjustments can be made to the design in order to obtain parameters that are consistent with probabilities and that satisfies the legal requirements that are common in credit scoring (De Waal et al, 2005). In particular, a configuration based on linear transfer functions and softmax (logistic) output functions is used. In this case, a probability matrix of dimension $\left(\left|V_{\text {past }}\right|+1\right) \times 3$ is obtained. The classification function for each $k, k \in\{P, C, W\}$ is:

$$
p_{k}(x)=\frac{\exp \left(\beta_{0}^{k}+\sum_{j=1}^{\left|V_{\text {past }}\right|} \beta_{j}^{k} x_{j}\right)}{\sum_{l \in\{P, C, W\}} \exp \left(\beta_{0}^{l}+\sum_{j=1}^{\left|V_{\text {past }}\right|} \beta_{j}^{l} x_{j}\right)}
$$

The results from both models are benchmarked against the results from a regular logistic regression, which delivers a unique set of parameters $\beta=\left(\beta_{0}, \ldots, \beta_{\left|V_{\text {pas }}\right|}\right)$ that construct probability $p(x)$ of being a defaulter in class $D=\{C, W\}$, a unique class. The expression of $p(x)$ is:

$$
p(x)=\frac{1}{1+\exp \left(-\beta_{0}-\sum_{j=1}^{\left|V_{\text {past }}\right|} \beta_{j} x_{j}\right)}
$$

In order to construct the experiments, a balanced sample was taken from database $X_{\text {past }}$, selecting 14286 cases representing the three classes. Then 10-by-10 cross-validation was applied to the dataset to obtain the parameters and their deviations (Hastie et al, 2009). For training the neural network, an additional step-extracting one of the folds of the cross-validation set to search for the optimal parameter configuration - was taken, a crucial step in order to get useful results in these types of statistical models (Zhang, 2007). Since the training is performed 100 times, a set of 100 results is obtained for both the parameters and the performances of the models. In all tables that are presented the 'mean \pm std. deviation' of these results is reported.

The results for the different models can be seen in Table 4 . The results displayed are aggregated, that is, the obtained probabilities are transformed into defaulters and non-defaulters, which is performed by simple addition of the probabilities of default. This is done in order to facilitate comparison with the binomial model, which differentiates between $P$ and $D$. One could combine the scores in other ways (Zhu et al, 2001), but we believe that the direct methodology is the obvious approach in this case. This comparison also takes into account the fact that the final goal is to have a better discrimination between these two classes, defaulters and non-defaulters. The results obtained are more clearly viewed when the Area Under the Curve (AUC) is considered and the Receiver Operator Characteristics (ROC) curves are plotted. Table 4 shows the AUC that is obtained for each model, and Figure 1 displays the comparison between the models. The proposed methodologies obtain results that are $5-7 \%$ better than the logistic regression model. The AUC of the two models with the two types of defaulters are statistically insignificant in value, but both are superior to the logistic regression. The ROC curve also shows

Table 4 AUC for the three models

\begin{tabular}{lc}
\hline Model & AUC (defaulters) \\
\hline Logistic regression (w/o defaulter diff.) & $0.6275 \pm 0.017$ \\
Multinomial logistic regression (w/ defaulter diff.) & $0.6678 \pm 0.004$ \\
Neural networks (w/ defaulter diff.) & $0.6660 \pm 0.004$ \\
\hline
\end{tabular}




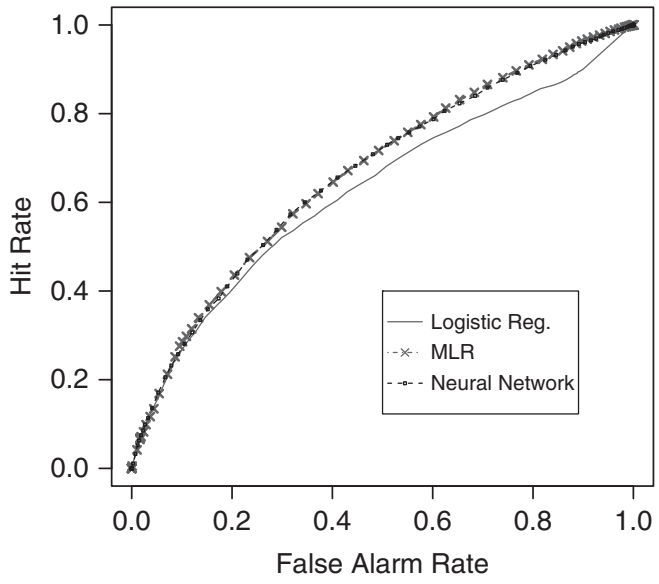

Figure 1 ROC curves for the three proposed models.

that the multinomial models are better at identifying defaulters in the upper range of scores, showing a significant difference when more than $30 \%$ of the true positives have been detected. This suggests the increase can be attributed to the improvement in discriminating between the two types of defaulters. Since the dataset was obtained given a very low rejection rate, and that the increase in discrimination capacity comes from a better differentiation of defaulters, the improvement that the methodology brings is subject to the proportion of cases in each class that any potential user has; a bank better at filtering bad borrowers (class $W$ ) might not see such a large increase in default prediction, whereas an institution with lower standards (as the one used in this example) will observe better results.

In Tables 5, 6a and $6 \mathrm{~b}$ the parameters from the experiments can be observed. The first conclusion that can be drawn from the parameters is their low standard deviation, hinting to very stable solutions. The improvement in classification can be explained by those variables whose $\beta$ parameters for good payers lies in between the respective parameters for classes $C$ and $W$ as happens, for example, with the variable 'Tenant'. This variable is not significant in the binomial logistic regression model. It is though in the other two models, with both models showing that being a tenant is a characteristic present for customers who do not have the capacity to repay, and that it is a neutral characteristic for good payers. Owning a house, in turn, is not a characteristic of customers with lack of willingness to pay. Another example is the variable 'Guarantor'. Having a guarantor means one is more likely to want to pay, reflected by the negative coefficient from class $W$. Of course, the lender may create this effect by only requiring guarantors from borrowers who the lender thinks may not be able to repay.

Borrowers in class $W$ usually ask for longer terms, and adding this to the smaller amounts that they borrow implies an interesting behaviour: a customer who may default because he or she does not want to pay will borrow a small amount of money repaid with small instalments, contrasted with borrowers of class $C$ who are prone to a greater risk given that the amount in each instalment is larger.
Table $5 \beta$ parameters obtained from neural network training with defaulter differentiation

\begin{tabular}{lcrr}
\hline Variable & $P$ & $C$ & \multicolumn{1}{c}{$W$} \\
\hline Activity_A & $0.06 \pm 0.01$ & $-0.29 \pm 0.03$ & $0.16 \pm 0.02$ \\
Activity_B & $0.05 \pm 0.01$ & $-0.16 \pm 0.02$ & $0.09 \pm 0.01$ \\
Owner & $0 \pm 0$ & $-0.1 \pm 0.62$ & $-0.07 \pm 0.01$ \\
Tenant & $-0.09 \pm 0.01$ & $0.28 \pm 0.03$ & $-0.21 \pm 0.02$ \\
Middle_Own & $-0.09 \pm 0.01$ & $0.21 \pm 0.03$ & $-0.12 \pm 0.02$ \\
No_Property & $-0.15 \pm 0.02$ & $0.07 \pm 0.03$ & $0.11 \pm 0.01$ \\
One_Property & $-0.05 \pm 0.01$ & $0.01 \pm 0.01$ & $0.04 \pm 0.01$ \\
Region_1 & $-0.17 \pm 0.02$ & $0.16 \pm 0.03$ & $-0.03 \pm 0.01$ \\
Region_2 & $0.12 \pm 0.02$ & $-0.21 \pm 0.03$ & $0 \pm 0$ \\
Guarantor & $0.06 \pm 0.01$ & $0.09 \pm 0.01$ & $-0.15 \pm 0.02$ \\
Term & $-0.44 \pm 0.18$ & $-0.45 \pm 0.46$ & $1.12 \pm 0.18$ \\
Age & $7.31 \pm 1.13$ & $3.91 \pm 1.88$ & $-11.99 \pm 1.93$ \\
\hline
\end{tabular}

Table $6 \beta$ parameters obtained from logistic regression models, with and without defaulter differentiation

(a) Multinomial regression

\begin{tabular}{lrr}
\hline Variable & \multicolumn{1}{c}{$P$} & \multicolumn{1}{c}{$C$} \\
\hline Activity_A & $0.36 \pm 0.04$ & $-0.4 \pm 0.05$ \\
Activity_B & $-0.25 \pm 0.02$ & $-1.37 \pm 0.03$ \\
Owner & $-0.08 \pm 0.02$ & $-0.71 \pm 0.02$ \\
Tenant & $0.18 \pm 0.02$ & $0.55 \pm 0.03$ \\
Middle_Own & $0.31 \pm 0.03$ & $1.46 \pm 0.03$ \\
No_Property & $0.03 \pm 0.04$ & $1.01 \pm 0.04$ \\
One_Property & $-0.76 \pm 0.01$ & $-0.14 \pm 0.02$ \\
Region_1 & $-0.25 \pm 0.03$ & $-0.11 \pm 0.03$ \\
Region_2 & $-0.43 \pm 0.03$ & $0.55 \pm 0.02$ \\
Guarantor & $0.29 \pm 0.02$ & $-0.69 \pm 0.03$ \\
Term & $0.62 \pm 0.02$ & $0.72 \pm 0.03$ \\
Age & $-0.41 \pm 0.05$ & $-0.29 \pm 0.06$ \\
\hline
\end{tabular}

(b) Binomial regression

\begin{tabular}{lr}
\hline Variable & \multicolumn{1}{c}{ Defaulter } \\
\hline Activity_A & $0.33 \pm 0.04$ \\
Activity_B & $-0.29 \pm 0.02$ \\
Owner & $-0.27 \pm 0.02$ \\
Tenant & $0 \pm 0.02$ \\
Middle_Own & $0.39 \pm 0.03$ \\
No_Property & $0.39 \pm 0.03$ \\
One_Property & $0.69 \pm 0.02$ \\
Region_1 & $0.2 \pm 0.02$ \\
Region_2 & $0.73 \pm 0.03$ \\
Guarantor & $-0.56 \pm 0.03$ \\
Term & $-0.25 \pm 0.03$ \\
Age & $0.19 \pm 0.04$ \\
\hline
\end{tabular}

\section{Conclusions and future work}

In this paper, we introduced a novel methodology that provides opportunities to improve credit scoring systems. Its key ideas are to describe the behaviour of borrowers using economic modelling, which subsequently leads to 
restrictions that via constrained clustering reveal differences among defaulters, a pattern that to the best of our knowledge has not been exploited so far. The combined applications of data mining, statistical analysis, and game theory are being used in a large number of fields, and we present how they also show promise in financial analysis, where the behaviour of different agents is key.

The results show a significant improvement over the classic methodology, with a $6 \%$ average increase in discrimination capacity, which can be fairly significant considering the amounts involved in consumer lending. However, an even greater contribution is identifying the two types of defaulters. Their characteristics bring interesting information about how the types of defaulters behave and can be detected. That knowledge can be used to improve the basic requirements for loans, or to detect risk segments of the portfolios in order to improve collecting campaigns. Better understanding of these segments also has the potential to lower the overall risk present in the portfolios, with large potential savings.

The procedure in this work focused on applying semisupervised techniques to identify unknown patterns, aided by a simple decision process extracted from economic modelling. This research can be extended by designing more complex games that reflect the decision processes that defaulters make in greater detail, for example focusing on capturing irrational behaviours using behavioural economics. Some results in this setting can be seen for example in the work of Benton et al (2007), among others. A second approach would be to calculate a general equilibrium, using an endogenous decision process rather than an exogenous differentiation of defaulters. In both cases a careful estimation of a set of constraints that would allow for knowledge discovery techniques to be applied would have to be performed, since a complex economic model will lead to equilibria that are not easily transformed to constraints as was the case in our work. Another extension would be, if possible, to obtain the actual reason for default of a given dataset, even if it is a small fraction of defaulters, and developing a more empirical model given this new data. The model can then be adjusted to incorporate the new information, hopefully leading to stronger results.

Finally, the main conclusion of this work is that traditional credit scoring can, and should, be improved by the use of more sophisticated techniques. The current developments in the fields of statistics, economics, and behavioural analysis are powerful tools that are available for researchers and practitioners.

Acknowledgements - The first author acknowledges CONICYT for the grants that support this work (AT-24110006, NAC-DOC: 21090573) and the $\mathrm{PhD}$ in Engineering Systems, Universidad de Chile. All authors acknowledge the support of the institution that provided the data. The work reported in this paper has been partially funded by the Institute of Complex Engineering Systems (ICM: P-05-004-F, CONICYT: FBO16) and the Finance Center of the Department of Industrial Engineering, Universidad de Chile, with the support of Bank Bci.

\section{References}

Alary D and Gollier C (2004). Debt contract, strategic default, and optimal penalties with judgement errors. Annals of Economics and Finance 5(2): 357-372.

Anderson R (2007). The Credit Scoring Toolkit. Oxford University Press: New York.

Baesens B, Van Gestel T, Viaene S, Stepanova M, Suykens J and Vanthienen J (2003). Benchmarking state-of-the-art classification algorithms for credit scoring. Journal of the Operational Research Society 54(6): 627-636.

Baesens B, Van Gestel T, Stepanova M and Vanthienen J (2005). Neural network survival analysis for personal loan data. Jounal of the Operational Research Society 56(9): 1089-1098.

Bard JF and Jarrah A (2009). Large-scale constrained clustering for rationalizing pickup and delivery operations. Transportation Research Part B: Methodological 43(5): 542-561.

Basu S, Bilenko M and Mooney RJ (2004). A probabilistic framework for semi-supervised clustering. In: Proceedings of the tenth ACM SIGKDD international conference on Knowledge discovery and data mining, KDD '04, ACM: New York, pp 59-68.

Basu S, Davidson I and Wagstaff K (2008). Constrained Clustering: Advances in Algorithms, Theory, and Applications. Chapman \& Hall/ CRC: Florida, USA.

Benton M, Meier S and Sprenger C (2007). Overborrowing and undersaving: Lessons and policy implications from research in behavioral economics. Public and Community Affairs Discussion Papers 2007-4, Federal Reserve Bank of Boston.

Benzion U, Rapoport A and Yagil J (1989). Discount rates inferred from decisions: An experimental study. Management Science 35(3): 270-284.

Block-Lieb S and Janger EJ (2006). The myth of the rational borrower: Rationality, behaviorism, and the misguided 'reform' of bankruptcy law. Texas Law Review 84(6): 1481-1565.

Bravo C and Weber R (2011). Semi-supervised constrained clustering with cluster outlier filtering. In: CS Martin and S-W Kim (eds.) Progress in Pattern Recognition, Image Analysis, Computer Vision, and Applications, Lecture Notes in Computer Science Vol. 7042, Springer-Verlag: Berlin/Heidelberg, pp 347-354.

Bravo C, Maldonado S and Weber R (2013). Granting and managing loans for micro-entrepreneurs: New developments and practical experiences. European Journal of Operational Research 227(2): 358-366.

Burks SV, Carpenter JP, Goette L and Rustichini A (2008). Cognitive skills explain economic preferences, strategic behavior, and job attachment. IZA Discussion Papers 3609, Institute for the Study of Labor (IZA).

Chabris CF, Laibson D, Morris CL, Schuldt JP and Taubinsky D (2008). Individual laboratory-measured discount rates predict field behavior. NBER Working Paper No. 14270, 2008. http://www.nber.org/papers/ w14270, accessed 10 February 2012.

Davidson I and Ravi SS (2005). Clustering with constraints: Feasibility issues and the k-means algorithm. In: Proceedings of the SIAM International Conference on Data Mining, SDM 2005; April 21-23, New Port Beach, CA, pp 138-149.

De Waal DA, Du Toit JV and De La Rey T (2005). An investigation into the use of generalized additive neural networks in credit scoring. In: Proceedings of IX Credit Scoring \& Credit Control Conference, Pollock Halls, University of Edinburgh, Scotland, 26-28 August, 2009, pp 1-10.

Dogan H and Guzelis C (2006). Gradient networks for clustering. In: Gknar IC and Sevgi L (eds). Complex Computing-Networks, Volume 104 of Springer Proceedings Physics; Springer Berlin Heidelberg, pp 275-278.

Fudenberg D and Tirole J (1991). Game Theory. MIT Press: Massachusetts, USA. 
Green L, Fry AF and Myerson J (1994). Discounting of delayed rewards: A life-span comparison. Psychological Science 5(1): $33-36$.

Guiso L, Sapienza P and Zingales L (2010). The determinants of attitudes towards strategic default on mortgages. Economics Working Papers ECO2010/31, European University Institute.

Hastie T, Tibshirani R and Friedman J (2009). The Elements of Statistical Learning: Data Mining, Inference, and Prediction. 2nd edn. Springer: New York, USA.

Hosmer D and Lemeshow H (2000). Applied Logistic Regression. John Wiley \& Sons: Massachusetts, USA.

Jokivuolle E and Peura S (2000). A model for estimating recovery rates and collateral haircuts for bank loans. Research Discussion Papers 2/ 2000, Bank of Finland.

Levy M and Sandler M (2008). Structural segmentation of musical audio by constrained clustering. IEEE Transactions on Audio, Speech, and Language Processing 16(2): 318-326.

L'Huillier G, Weber R and Figueroa N (2009). Online phishing classification using adversarial data mining and signaling games. SIGKDD Exploration Newsletter 11: 92-99.

Lin S, Ansell J and Andreeva G (2011). Predicting default of a small business using different definitions of financial distress. The Journal of the Operational Research Society 63(4): 539-548.

Littwin A (2007). Beyond usury: A study of credit-card use and preference among low-income consumers. Texas Law Review 86(3): 451-506.

Moffat PG (2005). Hurdle models of loan default. The Journal of the Operational Research Society 56(9): 1063-1071.

Patil G, Modarres R, Myers W and Patankar P (2006). Spatially constrained clustering and upper level set scan hotspot detection in surveillance geoinformatics. Environmental and Ecological Statistics 13(4): 365-377.
Siddiqi N (2006). Credit Risk Scorecards: Developing and Implementing Intelligent Credit Scoring. John Wiley and Sons: New Jersey, USA.

Stiglitz JE and Weiss A (1981). Credit rationing in markets with imperfect information. American Economic Review 71(3): 393-410.

Thomas LC (2000). A survey of credit and behavioural scoring: forecasting financial risk of lending to consumers. International Journal of Forecasting 16(2): 149-172.

Thomas LC, Crook J and Edelman D (2002). Credit Scoring and Its Applications. SIAM: Philadelphia, USA.

Vatsa V, Sural S and Majumdar A (2005). A game-theoretic approach to credit card fraud detection. In: Jajodia S and Mazumdar C (eds). Information Systems Security. Lecture Notes in Computer Science Vol. 3803. Springer-Verlag: Berlin/Heidelberg, pp 263-276.

Wang Y (2007). Combining data mining and game theory in manufacturing strategy analysis. Journal of Intelligent Manufacturing 18(4): $505-511$.

Wette HC (1983). Collateral in credit rationing in markets with imperfect information: Note. The American Economic Review 73(3): 442-445.

Yamashita S and Yoshiba T (2010). Analytical solution for expected loss of a collateralized loan: A square-root intensity process negatively correlated with collateral value. Discussion Paper 2010-E-10, Bank of Japan.

Zhang GP (2007). Avoiding pitfalls in neural network research. IEEE Transactions on Systems, Man, and Cybernetics, Part C 37(1): 3-16.

Zhu H, Beling PA and Overstreet GA (2001). A study in the combination of two consumer credit scores. The Journal of the Operational Research Society 52(9): 974-980.

Received 13 February 2012; accepted 25 March 2014 after two revisions 